JOURNAL OF

SYMPLECTIC GEOMETRY

Volume 11, Number 3, 475-488, 2013

\title{
ON THE HOFER GEOMETRY FOR WEAKLY EXACT LAGRANGIAN SUBMANIFOLDS
}

\author{
FroL ZAPOLSKY
}

We use spectral invariants in Lagrangian Floer theory in order to show that there exist isometric embeddings of normed linear spaces (finite or infinite-dimensional, depending on the case) into the space of Hamiltonian deformations of certain weakly exact Lagrangian submanifolds in tame symplectic manifolds. In addition to providing a new class of examples in which the Lagrangian Hofer metric can be computed explicitly, we refine and generalize some known results about it.

\section{Introduction and results}

Consider the following definition. Let $(W, \omega)$ be a symplectic manifold and let $L \subset W$ be a closed connected Lagrangian submanifold. Let $\mathcal{L}(L)$ be the space of all Lagrangian submanifolds of $W$ Hamiltonian isotopic to $L$. For $L^{\prime}, L^{\prime \prime} \in \mathcal{L}(L)$ we define

$$
\rho\left(L^{\prime}, L^{\prime \prime}\right)=\inf \left\{\int_{0}^{1} \operatorname{osc} H_{t} d t \mid \phi_{H}\left(L^{\prime}\right)=L^{\prime \prime}\right\}
$$

where $H_{t}$ is a compactly supported time-dependent Hamiltonian on $W$ and $\phi_{H}$ is the time-1 map of its flow. Chekanov [4] showed that if $J$-holomorphic curves in $W$ satisfy the maximum principle for a suitable class of almost complex structures $J$, then this quantity is a metric on $\mathcal{L}(L){ }^{1}$

Before stating our main result, we define the class of examples we deal with in this paper.

Definition 1.1. A closed connected Lagrangian submanifold $L \subset W$ is called weakly exact if $\left.\omega\right|_{\pi_{2}(W, L)}=0$. A pair $\left(L, L^{\prime}\right)$ of closed connected Lagrangian submanifolds of $W$ is called weakly exact if for any smooth $u: S^{1} \times[0,1] \rightarrow W$ with $u\left(S^{1} \times 0\right) \subset L, u\left(S^{1} \times 1\right) \subset L^{\prime}$ we have $\int u^{*} \omega=0$.

\footnotetext{
${ }^{1}$ Ibid., Chekanov gives an example of a symplectic manifold for which the condition on the behavior of holomorphic curves is not satisfied and for which $\rho$ is degenerate.
} 
Remark 1.2. Note that if $L \subset W$ is weakly exact, then so is $W$, that is $\omega$ vanishes on $\pi_{2}(W)$.

We assume for the rest of the paper that $W$ is connected and is tame in the sense of Biran-Cieliebak [3], that is it is either closed, or open and either admits an almost complex structure for which it is geometrically bounded (that is, tame in the ordinary sense of the word), or it is convex at infinity in the sense of Eliashberg-Gromov [5]. Also, we assume that all the almost complex structures $J$ are chosen so that $J$-holomorphic curves in $W$ are well-behaved (that is compactness holds) so that Floer homology can be defined. The main result is as follows.

Theorem 1.3. Let $L, L_{0}, \ldots, L_{k} \subset W$ be weakly exact Lagrangian submanifolds, such that each pair $\left(L, L_{j}\right)$ is weakly exact, and such that $L$ intersects each $L_{j}$ transversely at a single point, and $L_{j} \cap L_{j^{\prime}}=\varnothing$ for $j \neq j^{\prime}$. Then there is an isometric embedding $\left(\mathbb{R}^{k}, \mathrm{osc}_{0}\right) \rightarrow(\mathcal{L}(L), \rho)$.

Here osc $_{0}$ is a norm on $\mathbb{R}^{k}$ obtained as the restriction to $\mathbb{R}^{k}=0 \oplus \mathbb{R}^{k} \subset$ $\mathbb{R}^{k+1}$ of the oscillation osc : $\mathbb{R}^{k+1} \rightarrow[0, \infty)$ defined by $\operatorname{osc}\left(\tau_{0}, \ldots, \tau_{k}\right)=$ $\max _{j, j^{\prime}}\left(\tau_{j}-\tau_{j^{\prime}}\right)$.

Example 1.4. Examples of such manifolds are provided by the plumbing construction. Namely, let $L, L_{0}, \ldots, L_{k}$ be closed connected manifolds and consider their plumbing $W$ where the incidence graph is given by the tree with $L$ as the root and $L_{0}, \ldots, L_{k}$ as the leaves. We refer the reader to [1] for details of the plumbing construction. The resulting manifold is convex at infinity. Note that the symplectic manifold obtained in this way is exact, and so are the Lagrangians comprising its core.

The novelty of this result is threefold. First, as mentioned in [15], in case $W=T^{*} Q$, where $Q$ is closed and connected, there is an isometric embedding of $\left(C^{\infty}(Q) / \mathbb{R}\right.$, osc $)$ into $\mathcal{L}\left(\mathcal{O}_{Q}\right)$ where $\mathcal{O}_{Q} \subset T^{*} Q$ is the zero section. However, the whole cotangent bundle is needed even in order to show that $\mathcal{L}\left(\mathcal{O}_{Q}\right)$ has infinite diameter. In contrast, in our result the manifold $W$ may be a small neighborhood of the union $L \cup \bigcup_{j} L_{j}$, in particular, it may have finite volume or symplectic capacities. Intuitively, in the cotangent bundle, the zero section can be "moved" in different directions inside the space $\mathcal{L}\left(\mathcal{O}_{Q}\right)$ along the various cotangent fibers. In our situation $L$ is "moved" in different directions along the $L_{j}$. Next, as remarked in [8], sometimes one may pass to a covering space of the symplectic manifold and use the energy-capacity inequality to deduce results about the Hofer metric. In our situation, $W$ may well be simply connected, so this method will not apply. Thirdly, in [15] Usher constructed quasi-isometric embeddings of normed linear spaces into $\mathcal{L}$. Although in certain situations his embeddings are isometric, our theorem covers a new class of examples of such embeddings, in particular since $W$ may be non-compact. 
The next result covers some known cases, and also provides a new class of examples.

Theorem 1.5. Let $L, L^{\prime}$ be weakly exact Lagrangian submanifolds in $W$, such that the pair $\left(L, L^{\prime}\right)$ is weakly exact, and such that $L$ intersects $L^{\prime}$ transversely at a single point. If in addition $L^{\prime}$ admits a non-singular closed 1 -form, ${ }^{2}$ which vanishes on the kernel of the morphism $\pi_{1}\left(L^{\prime}\right) \rightarrow \pi_{1}(W)$ induced by inclusion, then there is an isometric embedding of $\left(C_{c}^{\infty}(0,1)\right.$, osc $)$ into $(\mathcal{L}(L), \rho)$.

Example 1.6. If $W$ is a surface, compact or not, and $L, L^{\prime}$ are a pair of noncontractible curves intersecting transversely at one point, then the theorem applies. This reproduces part of the result of Usher [15, Theorem 1.3]. As will be clear from the proof, when $W=\mathbb{T}^{2}$ and $L, L^{\prime}$ are two embedded noncontractible circles which transversely intersect at one point, there is actually an isometric embedding of $\left(C^{\infty}\left(S^{1}\right) / \mathbb{R}\right.$, osc) into $\mathcal{L}(L)$. More generally, if $W=\mathbb{T}^{2 n}$ and $L, L^{\prime}$ are two linear Lagrangian tori which intersect at a single point, the theorem applies, and as it is clear from the proof, there is an isometric embedding of $\left(C^{\infty}\left(\mathbb{T}^{n}\right) / \mathbb{R}\right.$, osc $)$ into $\mathcal{L}(L)$.

Example 1.7. This theorem can be applied to plumbings, as follows. If $L, L^{\prime}$ are two closed connected manifolds, we can form their single-intersection plumbing $W$; it follows from the plumbing construction that both $L, L^{\prime}$ are weakly exact, the pair $\left(L, L^{\prime}\right)$ is weakly exact and moreover the inclusion $L^{\prime} \rightarrow W$ induces an injection $\pi_{1}\left(L^{\prime}\right) \rightarrow \pi_{1}(W)$. Therefore, if $L^{\prime}$ admits a nonsingular closed 1-form, the theorem applies.

1.1. Preliminaries and notations. In the rest of the paper all manifolds are connected and all Lagrangian submanifolds are closed.

A Hamiltonian on $W$ is a function ${ }^{3} \quad H \in C_{c}^{\infty}([0,1] \times W)$. If $W$ is closed, then, unless stated otherwise, all Hamiltonians are normalized, that is $\int_{W} H_{t} \omega^{n}=0$ for all $t$. The Hamiltonian vector field of $H$ is defined via $\omega\left(X_{H_{t}}, \cdot\right)=-d H_{t}$. The flow $\phi_{H}^{t}$ of $H$ is defined by $\phi_{H}^{0}=\mathrm{id}, \frac{d \phi_{H}^{t}}{d t}=X_{H_{t}} \circ \phi_{H}^{t}$. We abbreviate $\phi_{H}=\phi_{H}^{1}$. The set of all time- 1 flows of all the Hamiltonians is a group $\mathcal{G}$, called the Hamiltonian group of $W$. When $L$ is a Lagrangian submanifold, the subgroup $\mathcal{G}_{L} \subset \mathcal{G}$ consists of all the diffeomorphisms fixing $L$ as a set.

When $K$ is a Hamiltonian, the reverse Hamiltonian $\bar{K}$ is defined via $\bar{K}(t, x)=-K(1-t, x)$. It generates the reverse isotopy $\phi_{\bar{K}}^{t}=\phi_{K}^{1-t} \phi_{K}^{-1}$. If $\gamma:[0,1] \rightarrow W$ is a path, the reverse path $\bar{\gamma}$ is defined by $\bar{\gamma}(t)=\gamma(1-t)$.

\footnotetext{
${ }^{2}$ This is equivalent to $L^{\prime}$ fibering over $S^{1}$.

${ }^{3}$ When we concatenate Hamiltonians, we obtain functions in $C_{c}^{\infty}([0,2] \times W)$. The necessary adjustments are left to the reader.
} 
We will need to concatenate Hamiltonians and paths. If $z, z^{\prime}:[0,1] \rightarrow Z$ are paths, where $Z$ is a space (in this paper $Z$ can be $W, \mathcal{G}$, or $C_{c}^{\infty}(W)$ ) we let

$$
\left(z \sharp z^{\prime}\right)(t)= \begin{cases}z(t), & t \leq 1, \\ z^{\prime}(t-1), & t>1 .\end{cases}
$$

This is a path defined over the interval [0,2]. It is continuous if $z(1)=z^{\prime}(0)$ and smooth if all the derivatives of $z$ at 1 coincide with those of $z^{\prime}$ at 0 . A particularly important case is when we are given two Hamiltonians $H, K$ on $W$ and we need to concatenate them. There is a procedure, called smoothing, which allows us to do this for any pair of Hamiltonians, see for example [11]. Briefly, one chooses a function $f:[0,1] \rightarrow[0,1]$ which is smooth, monotone, and equals to 0 near 0 and to 1 near 1 . Put $H^{f}(t, x)=f^{\prime}(t) H(f(t), x)$. Then $H^{f}$ is also normalized, moreover it equals 0 for $t$ near 0,1 . Therefore the concatenation $H^{f} \sharp K^{g}$, where $g$ is another smoothing function like this, is always well-defined and smooth. Moreover, dynamical invariants of $H$ are left intact, namely, the action spectrum, the set of periodic orbits or orbits with given boundary conditions, and the spectral invariants. More details can be found in [11]. Below, whenever we concatenate Hamiltonians, it is implicitly assumed that they have been previously smoothed.

We will also use various action functionals. Fix a Hamiltonian $H$. Let $\gamma$ be a smooth contractible loop in $W$ and let $u$ be a contracting disc, that is a map $u: D^{2} \rightarrow W$ such that $\gamma=\left.u\right|_{\partial D}$. Define

$$
\mathcal{A}_{H}(\gamma)=\int_{0}^{1} H_{t}(\gamma(t)) d t-\int_{D^{2}} u^{*} \omega .
$$

If $\left.\omega\right|_{\pi_{2}(W)}=0$, then this is independent of $u$.

If $\gamma$ is a smooth path with endpoints on a Lagrangian $L$, we call it contractible for brevity, if $[\gamma]$ is the trivial element in $\pi_{1}(W, L)$. Let $u$ be a contraction of $\gamma$ into $L$, that is $u: D_{+}^{2} \rightarrow M$, where $D_{+}^{2}=\{z \in \mathbb{C}|| z \mid \leq 1$, $\operatorname{Im} z \geq 0\}$, with $u(\exp (\pi i t))=\gamma(t)$ and $u(t) \in L$ for $t \in[-1,1]$. Then put

$$
\mathcal{A}_{H}^{L}(\gamma)=\int_{0}^{1} H_{t}(\gamma(t)) d t-\int_{D_{+}^{2}} u^{*} \omega
$$

When $\left.\omega\right|_{\pi_{2}(W, L)}=0$, this is independent of $u$.

1.2. Proofs of main results. After the existence of spectral invariants in Lagrangian Floer homology has been established in the next section, the proof of main results is elementary, therefore we provide it here.

Before passing to the proof, we describe the construction of the so-called action homomorphism. It is instrumental for the methods of the present paper. Let $L \subset W$ be a weakly exact Lagrangian submanifold. If $\alpha \in \mathcal{G}_{L}$ and $H$ is a Hamiltonian generating $\alpha$, we put $\gamma_{q}(t)=\phi_{H}^{t}(q)$ for $q \in L$. We have 
Proposition 1.8. The Hamiltonian chords $\gamma_{q}$ are all contractible and the action $\mathcal{A}_{H}^{L}\left(\gamma_{q}\right)$ only depends on $\alpha$. The map $\mathcal{A}^{L}: \mathcal{G}_{L} \rightarrow \mathbb{R}$, thus defined is a homomorphism. Moreover, if $H$ is a time-dependent Hamiltonian with $\left.H_{t}\right|_{L}=c \in \mathbb{R}$ for all $t$, we have $\mathcal{A}^{L}\left(\phi_{H}\right)=c$.

The proof is given in subsection 2.2. We call this $\mathcal{A}^{L}$ the action homomorphism (associated to $L$ ).

Let us now formulate the properties of spectral invariants necessary for the proof of the main results.

Theorem 1.9. Let $L, L^{\prime} \subset W$ be weakly exact Lagrangian submanifolds, which intersect transversely at a single point, and such that the pair $\left(L, L^{\prime}\right)$ is weakly exact. Then there is a function $\ell\left(\cdot: L, L^{\prime}\right): \mathcal{G} \rightarrow \mathbb{R}$ which satisfies:

(i) $\int_{0}^{1} \min \left(F_{t}-G_{t}\right) d t \leq \ell\left(\phi_{F}: L, L^{\prime}\right)-\ell\left(\phi_{G}: L, L^{\prime}\right) \leq \int_{0}^{1} \max \left(F_{t}-G_{t}\right) d t$;

(ii) for $\alpha \in \mathcal{G}_{L^{\prime}}$ we have $\ell\left(\alpha \phi: L, L^{\prime}\right)=\ell\left(\phi: L, L^{\prime}\right)+\mathcal{A}^{L^{\prime}}(\alpha)$;

(iii) for $\beta \in \mathcal{G}_{L}$ we have $\ell\left(\beta: L, L^{\prime}\right)=\mathcal{A}^{L}(\beta)$; if $H$ is a Hamiltonian with $\left.H_{t}\right|_{L}=c \in \mathbb{R}$, then $\ell\left(\phi_{H}: L, L^{\prime}\right)=\mathcal{A}^{L}\left(\phi_{H}\right)=c$.

Theorem 1.9 follows from the more general result, theorem 2.6, to whose proof is dedicated most of Section 2. Now we are ready to prove the main results. In order to compute Hofer distances between various Lagrangians, lower and upper bounds need to be established. Upper bounds are obtained via the oscillation of certain Hamiltonians and are elementary. The nontrivial part is to prove lower bounds. Since the technical idea behind the existence of such lower bounds is the same for both of the theorems, and is interesting on its own, we formulate it as a separate lemma.

Lemma 1.10. Let $Q, Q^{\prime}, Q^{\prime \prime} \subset W$ be weakly exact Lagrangian submanifolds, such that $Q, Q^{\prime \prime}$ are disjoint, $Q \cap Q^{\prime}$ and $Q^{\prime \prime} \cap Q^{\prime}$ are both transverse intersections which are single points, and such that both the pairs $\left(Q, Q^{\prime}\right)$, $\left(Q^{\prime \prime}, Q^{\prime}\right)$ are weakly exact. If $H$ is a Hamiltonian which satisfies $\left.H\right|_{Q}=c$ and $\left.H\right|_{Q^{\prime \prime}}=c^{\prime \prime}$, where $c, c^{\prime \prime} \in \mathbb{R}$, then $\rho\left(\phi_{H}\left(Q^{\prime}\right), Q^{\prime}\right) \geq c-c^{\prime \prime}$.

Proof. Abbreviate $\phi=\phi_{H}$. Theorem 1.9 implies that $\ell\left(\phi: Q, Q^{\prime}\right)=c$. Now let $\alpha \in \mathcal{G}_{Q^{\prime}}$ and let $G$ be a Hamiltonian generating $\alpha \phi$. It follows that

$$
\int_{0}^{1} \max G_{t} d t \geq \ell\left(\alpha \phi: Q, Q^{\prime}\right)=\ell\left(\phi: Q, Q^{\prime}\right)+\mathcal{A}^{Q^{\prime}}(\alpha)=c+\mathcal{A}^{Q^{\prime}}(\alpha) .
$$

Analogously, $\ell\left(\phi: Q^{\prime \prime}, Q^{\prime}\right)=c^{\prime \prime}$, and also

$$
-\int_{0}^{1} \min G_{t} d t \geq-\ell\left(\alpha \phi: Q^{\prime \prime}, Q^{\prime}\right)=-c^{\prime \prime}-\mathcal{A}^{Q^{\prime}}(\alpha) .
$$

The above two inequalities added together imply

$$
\int_{0}^{1} \operatorname{osc} G_{t} d t \geq c-c^{\prime \prime}
$$


Taking infimum over $\alpha \in \mathcal{G}_{Q^{\prime}}$, we obtain

$$
c-c^{\prime \prime} \leq \rho\left(\phi\left(Q^{\prime}\right), Q^{\prime}\right) .
$$

Proof of Theorems 1.3 and 1.5. Let us first prove theorem 1.3. Recall that we have the Lagrangians $L, L_{0}, \ldots, L_{k}$. For $j=1, \ldots, k$ let $H_{j} \in C_{c}^{\infty}$ $(W,[0,1])$ be an autonomous Hamiltonian taking the value 1 on $L_{j}$, and the value 0 on all $L_{j^{\prime}}$ for $j^{\prime}=0, \ldots, k, j^{\prime} \neq j$, such that the supports of $H_{j}$ are all pairwise disjoint. For $\tau=\left(\tau_{1}, \ldots, \tau_{k}\right) \in \mathbb{R}^{k}$ let $H_{\tau}=\sum_{j} \tau_{j} H_{j}$. Put $L_{\tau}=\phi_{H_{\tau}}(L)$. We claim that

$$
\rho\left(L_{\tau}, L_{\tau^{\prime}}\right)=\operatorname{osc}_{0}\left(\tau-\tau^{\prime}\right)
$$

which is another way of saying that $\left(\mathbb{R}^{k}, \operatorname{osc}_{0}\right) \rightarrow(\mathcal{L}(L), \rho), \tau \mapsto L_{\tau}$, is an isometric embedding. This will imply the assertion of the theorem.

Let us prove this. Abbreviate $\phi_{\tau}=\phi_{H_{\tau}}$ and note that

$$
\rho\left(L_{\tau}, L_{\tau^{\prime}}\right)=\rho\left(\phi_{\tau}(L), \phi_{\tau^{\prime}}(L)\right)=\rho\left(\phi_{\tau-\tau^{\prime}}(L), L\right) .
$$

Therefore it is enough to show that

$$
\rho\left(L_{\tau}, L\right)=\operatorname{osc}_{0}(\tau)
$$

First,

$$
\rho\left(L_{\tau}, L\right)=\rho\left(\phi_{\tau}(L), L\right) \leq \operatorname{osc} H_{\tau}
$$

It follows from the definition of $H_{\tau}$ that $\operatorname{osc} H_{\tau}=\operatorname{osc}_{0}(\tau)$. On the other hand, lemma 1.10 with $Q^{\prime}=L, Q=L_{j}, Q^{\prime \prime}=L_{j^{\prime}}$, and $H=H_{\tau}$, implies

$$
\rho\left(L_{\tau}, L\right)=\rho\left(\phi_{\tau}(L), L\right) \geq \tau_{j}-\tau_{j^{\prime}}
$$

for all $j, j^{\prime}=0, \ldots, k$, where $\tau_{0}:=0$. Taking now the maximum over $j, j^{\prime}$ results in

$$
\operatorname{osc}_{0}(\tau)=\max _{j, j^{\prime}=0, \ldots, k}\left(\tau_{j}-\tau_{j}^{\prime}\right) \leq \rho\left(L_{\tau}, L\right) \leq \operatorname{osc}_{0}(\tau),
$$

which finishes the proof of Theorem 1.3.

Let us now prove theorem 1.5. Recall that we have two Lagrangians $L, L^{\prime}$. Let $\eta$ be a nonsingular closed 1 -form on $L^{\prime}$. Identify a Weinstein neighborhood $U$ of $L^{\prime}$ with a neighborhood of the zero section in $T^{*} L^{\prime}$, such that $L \cap U=T_{q_{0}}^{*} L^{\prime} \cap U$. Without loss of generality we assume that $U$ is the unit disc cotangent bundle of $L^{\prime}$ with respect to some auxiliary Riemannian metric on $L^{\prime}$. If necessary, scale $\eta$ so that its graph is contained in $U$. We let $L_{\tau}^{\prime}$ be the Lagrangian in $W$ corresponding to the graph of $\tau \eta$ inside $U$, where $\tau \in[0,1]$. It follows from the assumption that $\eta$ vanish on $\operatorname{ker}\left(\pi_{1}\left(L^{\prime}\right) \rightarrow \pi_{1}(W)\right)$ that $L_{\tau}^{\prime}$ is weakly exact and that the pair $\left(L_{\tau}^{\prime}, L\right)$ is weakly exact, and moreover $L \cap L_{\tau}^{\prime}$ is a single transverse intersection point. 
Choose a function $H \in C_{c}^{\infty}(U)$ such that $\left.H\right|_{L_{\tau}^{\prime}}=\tau$ for $\tau \in[0,1]$. Extend $H$ by zero to $W$. Define a map $C_{c}^{\infty}(0,1) \rightarrow \mathcal{L}(L)$ via $f \mapsto L_{f}:=\phi_{f}(L)$ where $\phi_{f}$ is the time- 1 map of the Hamiltonian $H_{f}=f \circ H$. We claim that

$$
\rho\left(L_{f}, L_{f^{\prime}}\right)=\operatorname{osc}\left(f-f^{\prime}\right)
$$

which is what the assertion we want to establish says. As in the first part, it is enough to show that $\rho\left(L_{f}, L\right)=\operatorname{osc} f$. On the one hand, we have

$$
\rho\left(L_{f}, L\right)=\rho\left(\phi_{f}(L), L\right) \leq \operatorname{osc} H_{f}=\operatorname{osc} f .
$$

On the other hand, if $\tau, \tau^{\prime} \in(0,1)$, lemma 1.10 applied with $Q^{\prime}=L, Q=L_{\tau}^{\prime}$, $Q^{\prime \prime}=L_{\tau^{\prime}}^{\prime}$, and $H=H_{f}$, implies that

$$
\rho\left(L_{f}, L\right) \geq f(\tau)-f\left(\tau^{\prime}\right) .
$$

It follows that

$$
\rho\left(L_{f}, L\right) \geq \max _{\tau, \tau^{\prime} \in(0,1)} f(\tau)-f\left(\tau^{\prime}\right)=\operatorname{osc} f,
$$

thereby completing the proof of the theorem.

1.3. Discussion. First, we would like to remark that the existence of spectral invariants implies results on the Hofer geometry of the Hamiltonian group itself. For $\phi, \psi \in \mathcal{G}$ we let

$$
\rho(\phi, \psi)=\inf \left\{\int_{0}^{1} \operatorname{osc} H_{t} d t \mid \phi \psi^{-1}=\phi_{H}\right\} .
$$

This is a metric on $\mathcal{G}$ (see for example [14]). It follows from [9] and the results of Section 2 that whenever $L \subset W$ is a weakly exact Lagrangian, its Floer homology $H F(H: L)$ with Hamiltonian perturbations can be used to produce spectral invariants $\ell(A, \cdot: L): \mathcal{G} \rightarrow \mathbb{R}$ relative to classes $A \in H F(L)=H(L)$. These satisfy properties analogous to those of the relative invariants introduced below. See also [11]. In particular, the following can be easily deduced.

Corollary 1.11. (i) If $L_{0}, L_{1}, \ldots, L_{k} \subset W$ are weakly exact Lagrangians, all pairwise disjoint, then there is an isometric embedding $\left(\mathbb{R}^{k}, \mathrm{osc}_{0}\right) \rightarrow(\mathcal{G}, \rho)$; (ii) if $L$ is a weakly exact Lagrangian that admits a non-singular closed 1 -form which vanishes on $\operatorname{ker}\left(\pi_{1}(L) \rightarrow \pi_{1}(W)\right)$, then there is an isometric embedding $\left(C_{c}^{\infty}(0,1)\right.$, osc $) \rightarrow(\mathcal{G}, \rho)$.

Secondly, we would like to point out that while the spectral invariants $\ell\left(\cdot: L, L^{\prime}\right): \mathcal{G} \rightarrow \mathbb{R}$ as in theorem 1.9 above are defined on the Hamiltonian group $\mathcal{G}$, if $L^{\prime \prime}$ is another weakly exact Lagrangian, disjoint from $L$ and intersecting $L^{\prime}$ transversely at one point, then the difference $\ell:=\ell\left(\cdot: L, L^{\prime}\right)-$ $\ell\left(\cdot: L^{\prime \prime}, L^{\prime}\right)$ in fact descends to $\mathcal{L}\left(L^{\prime}\right)$. The function thus obtained is in a sense (which can be made precise) a generalization of Viterbo's spectral invariants for Lagrangian submanifolds of cotangent bundles. This function 
$\ell: \mathcal{L}\left(L^{\prime}\right) \rightarrow \mathbb{R}$ is Lipschitz in the Hofer metric: $\ell\left(K, K^{\prime}\right) \leq \rho\left(K, K^{\prime}\right)$ for $K, K^{\prime} \in \mathcal{L}\left(L^{\prime}\right)$. Moreover, lower bounds on this function are obtained as in lemma 1.10 via the action homomorphism. Namely, if $\beta \in \mathcal{G}_{L}$ and $\beta^{\prime \prime} \in \mathcal{G}_{L^{\prime \prime}}$, then $\ell\left(\beta\left(L^{\prime}\right), \beta^{\prime \prime}\left(L^{\prime}\right)\right) \geq \mathcal{A}^{L}(\beta)-\mathcal{A}^{L^{\prime \prime}}\left(\beta^{\prime \prime}\right)$. This is what makes it useful in computing Hofer's metric on $\mathcal{L}\left(L^{\prime}\right)$.

\section{Spectral invariants in Lagrangian Floer theory}

In this section we assume that we are given two transversely intersecting weakly exact Lagrangians $L, L^{\prime} \subset W$, such that the pair $\left(L, L^{\prime}\right)$ is weakly exact. All homology is with $\mathbb{Z}_{2}$-coefficients and the count of moduli spaces is modulo 2 .

2.1. Definition and first properties. We begin with a brief sketch of the construction of the Floer homology of the pair $\left(L, L^{\prime}\right)$ with Hamiltonian perturbations. General references are $[\mathbf{6}, \mathbf{1 2}]$.

We fix an intersection point $q_{0} \in L \cap L^{\prime}$ once and for all and consider the connected component $\Omega$ of the constant path at $q_{0}$ in the space of smooth paths $\left\{\gamma:[0,1] \rightarrow W \mid \gamma(0) \in L, \gamma(1) \in L^{\prime}\right\}$. Since the pair $\left(L, L^{\prime}\right)$ is weakly exact, whenever $\gamma \in \Omega$ and $u$ is a homotopy from $q_{0}$ to $\gamma$, the integral

$$
\int u^{*} \omega
$$

is independent of $u$. Given a Hamiltonian $H$, we let $\mathcal{A}_{H}^{L, L^{\prime}}: \Omega \rightarrow \mathbb{R}$ be the action functional defined as

$$
\mathcal{A}_{H}^{L, L^{\prime}}(\gamma)=\int_{0}^{1} H_{t}(\gamma(t)) d t-\int u^{*} \omega
$$

for any homotopy $u$ from $q_{0}$ to $\gamma$. Its critical point set $\operatorname{Crit} \mathcal{A}_{H}^{L, L^{\prime}}$ consists precisely of those elements $\gamma \in \Omega$ which are Hamiltonian chords from $L$ to $L^{\prime}$, that is which satisfy $\dot{\gamma}(t)=X_{H_{t}}(\gamma(t))$. The map Crit $\mathcal{A}_{H}^{L, L^{\prime}} \rightarrow \phi_{H}(L) \cap L^{\prime}$, $\gamma \mapsto \gamma(1)$ is injective. We let $C F\left(H: L, L^{\prime}\right)$ be the $\mathbb{Z}_{2}$-vector space ${ }^{4}$ spanned by $\operatorname{Crit} \mathcal{A}_{H}^{L, L^{\prime}}$. We call $H$ regular if $\phi_{H}(L)$ intersects $L^{\prime}$ transversely. Regular Hamiltonians are generic. We choose $H$ regular, so that $\operatorname{Crit} \mathcal{A}_{H}^{L, L^{\prime}}$ is finite and $C F\left(H: L, L^{\prime}\right)$ is finite-dimensional.

Choose now a time-dependent compatible almost complex structure $J_{t}$ on $W$. This gives rise to an $L^{2}$-type metric on $\Omega$ and the negative gradient equation for $\mathcal{A}_{H}^{L, L^{\prime}}$ translates into Floer's PDE

$$
\frac{\partial u}{\partial s}+J_{t}(u)\left(\frac{\partial u}{\partial t}-X_{H_{t}}(u)\right)=0
$$

\footnotetext{
${ }^{4}$ This vector space is ungraded since there is no requirement on Maslov indices.
} 
for $u: \mathbb{R} \times[0,1] \rightarrow W$ satisfying the boundary conditions $u(\mathbb{R} \times 0) \subset L$, $u(\mathbb{R} \times 1) \subset L^{\prime}$. For $\gamma_{ \pm} \in \operatorname{Crit} \mathcal{A}_{H}^{L, L^{\prime}}$ we let $\widehat{\mathcal{M}}\left(\gamma_{-}, \gamma_{+} ; H, J\right)$ be the space of solutions of this PDE subject to the asymptotic conditions $u( \pm \infty, \cdot)=\gamma_{ \pm}$. For a generic choice of $J$ this is a finite-dimensional smooth manifold. Moreover, note that if $u \in \widehat{\mathcal{M}}\left(\gamma_{-}, \gamma_{+} ; H, J\right)$ then

$$
E(u):=\int_{\mathbb{R} \times[0,1]}\left|\frac{\partial u}{\partial s}\right|^{2} d s d t=\mathcal{A}_{H}^{L, L^{\prime}}\left(\gamma_{-}\right)-\mathcal{A}_{H}^{L, L^{\prime}}\left(\gamma_{+}\right) .
$$

Also note that $E(u) \geq 0$ with equality if and only if $u$ is a constant map. In case $\gamma_{-} \neq \gamma_{+}$we let $\mathcal{M}\left(\gamma_{-}, \gamma_{+} ; H, J\right)$ be the quotient by the natural action of $\mathbb{R}$. We also put $\mathcal{M}\left(\gamma_{-}, \gamma_{-} ; H, J\right)=\varnothing$.

The Floer boundary operator on $C F\left(H: L, L^{\prime}\right)$ is given on generators by

$$
\partial \gamma_{-}=\sum_{\gamma_{+}: \operatorname{dim} \mathcal{M}\left(\gamma_{-}, \gamma_{+} ; H, J\right)=0} \# \mathcal{M}\left(\gamma_{-}, \gamma_{+} ; H, J\right) \gamma_{+} .
$$

It is a standard fact that $\partial^{2}=0$ and we let $H F\left(H: L, L^{\prime}\right)$ be the corresponding homology. We omit the almost complex structure from the notation since neither the Floer homology nor the spectral invariants which we will introduce shortly depend on it.

Since $L, L^{\prime}$ intersect transversely, the zero Hamiltonian is regular. In this case $H F\left(0: L, L^{\prime}\right)$ is nothing but the (component at $q_{0}$ of the) usual Floer homology $H F\left(L, L^{\prime}\right)$ of the pair $\left(L, L^{\prime}\right)$. We record a particularly important special case:

Lemma 2.1. If $q_{0}$ is the only intersection point of $L, L^{\prime}$ then $H F(0$ : $\left.L, L^{\prime}\right)=\mathbb{Z}_{2} \cdot q_{0} \equiv \mathbb{Z}_{2}$.

Indeed, by definition $\mathcal{M}\left(q_{0}, q_{0} ; 0, J\right)=\varnothing$, therefore the boundary operator vanishes.

For any two regular Hamiltonians $H, H^{\prime}$ and any two regular almost complex structures $J, J^{\prime}$ there is a canonical continuation isomorphism $H F\left(H: L, L^{\prime}\right)=H F\left(H^{\prime}: L, L^{\prime}\right)=H F\left(L, L^{\prime}\right)$. Since the boundary operator counts negative gradient lines of the action functional, it follows that the subspace $C F^{a}\left(H: L, L^{\prime}\right) \subset C F\left(H: L, L^{\prime}\right)$ generated by the critical points of $\mathcal{A}_{H}$ of action $<a$ is a subcomplex. We let $i^{a}: H F^{a}\left(H: L, L^{\prime}\right) \rightarrow H F(H$ : $\left.L, L^{\prime}\right)=H F\left(L, L^{\prime}\right)$ be the morphism induced by the inclusion. The aforementioned continuation isomorphisms leave the maps $i^{a}$ intact if we only change $J$, therefore we omit it from the notation throughout. We are now in position to define the spectral invariants. For $A \in H F\left(L, L^{\prime}\right)-\{0\}$ we let

$$
\ell\left(A, H: L, L^{\prime}\right)=\inf \left\{a \mid A \in \operatorname{im} i^{a}\right\} .
$$

From the definition it follows that the spectral invariants are spectral, that is $\ell\left(A, H: L, L^{\prime}\right)$ belongs to the action spectrum $\operatorname{Spec}\left(H: L, L^{\prime}\right)=$ $\mathcal{A}_{H}^{L, L^{\prime}}\left(\operatorname{Crit} \mathcal{A}_{H}^{L, L^{\prime}}\right)$. 
We have defined spectral invariants for a regular Hamiltonian. Standard properties of the continuation isomorphisms imply the following:

$\int_{0}^{1} \min \left(H_{t}-H_{t}^{\prime}\right) d t \leq \ell\left(A, H: L, L^{\prime}\right)-\ell\left(A, H^{\prime}: L, L^{\prime}\right) \leq \int_{0}^{1} \max \left(H_{t}-H_{t}^{\prime}\right) d t$ for regular $H, H^{\prime}$. These inequalities allow us to define $\ell\left(A, G: L, L^{\prime}\right)$ for an arbitrary smooth Hamiltonian ${ }^{5} G$, in the standard manner. Using techniques similar to those of $[\mathbf{1 3}]$, we can show that these extended invariants are also spectral.

2.2. Hamiltonian loops and the action homomorphism. In order to be able to use spectral invariants in estimates on the Lagrangian Hofer distance, we need to show that they are defined on the Hamiltonian group, that is, that $\ell\left(A, H: L, L^{\prime}\right)$ in fact only depends on the time-1 map $\phi_{H}$. The first step is to show that the action of any periodic orbit of a Hamiltonian generating a loop in $\mathcal{G}$ is zero.

Let $G$ be a Hamiltonian generating a loop $^{6}$ in $\mathcal{G}$. If $W$ is open, since all the periodic orbits of $G$ are homotopic, it follows that all of them are contractible, because orbits outside the support of $G$ are just constant. It also follows that the action of these constant orbits is zero, and since the action is independent of the beginning of the orbit, all the actions of the periodic orbits vanish. Consider now the case of $W$ closed. The existence of Floer homology (see for example [7]) implies that every 1-periodic orbit of the form $t \mapsto \phi_{G}^{t}(z)$ is contractible. Moreover, it follows from [10, proposition 3.1 (i)] that the action of every such orbit is zero (remember that $G$ is normalized). We thus have

Lemma 2.2. Let $G$ be a Hamiltonian such that $\phi_{G}=\mathrm{id}$. Then any periodic orbit of the form $t \mapsto \phi_{G}^{t}(z)$, where $z \in W$, is contractible, its action is well-defined and equal to zero.

We can now establish the existence of the action homomorphism.

Proof or proposition 1.8. It follows from the existence of Floer homology for weakly exact Lagrangians (see for example [9]) that at least one of the chords $\gamma_{q}$ is contractible. Since all of them are homotopic, it follows that all of them are contractible. This implies that the actions $\mathcal{A}_{H}^{L}\left(\gamma_{q}\right)$ are well-defined and are all equal. It remains to show that this number is independent of $H$.

Put $\gamma_{q}^{H}(t)=\phi_{H}^{t}(q)$. Let $K$ be another Hamiltonian generating $\phi_{H}$, and let $\gamma_{q}^{K}(t)=\phi_{K}^{t}(q)$. We need to prove that $\mathcal{A}_{K}^{L}\left(\gamma_{q}^{K}\right)=\mathcal{A}_{H}^{L}\left(\gamma_{q}^{H}\right)$. Pick a contracting half-disc $u$ for $\gamma_{q}^{H}$. The concatenation $\gamma_{q}^{H} \sharp \gamma_{q}^{K}$ is a periodic orbit

\footnotetext{
${ }^{5}$ In fact, at the same price we can define them for arbitrary continuous Hamiltonians, however this will not be needed in this paper.

${ }^{6}$ In agreement with our convention, since $G$ needs to be time-periodic in order for what follows to make sense, we smooth it as described in Section 1.1.
} 
of the flow of the concatenated Hamiltonian $H \sharp \bar{K}$. Since this Hamiltonian generates the identity map, the above lemma implies that there is a contracting disc $v$ for $\gamma_{q}^{H} \sharp \overline{\gamma_{q}^{K}}$ and that

$$
\mathcal{A}_{H \sharp \bar{K}}\left(\gamma_{q}^{H} \sharp \overline{\gamma_{q}^{K}}\right)=0 .
$$

Writing out the action, we have

$$
\mathcal{A}_{H \sharp \bar{K}}\left(\gamma_{q}^{H} \sharp \overline{\gamma_{q}^{K}}\right)=\int_{0}^{2}(H \sharp \bar{K})_{t}\left(\left(\gamma_{q}^{H} \sharp \overline{\gamma_{q}^{K}}\right)(t)\right) d t-\int v^{*} \omega .
$$

Since $v$ provides a homotopy with fixed endpoints from $\gamma_{q}^{K}$ to $\gamma_{q}^{H}$, the concatenation $\bar{v} \sharp u$ is a contracting half-disc for $\gamma_{q}^{K}$ and therefore

$$
\begin{aligned}
\mathcal{A}_{H}^{L}\left(\gamma_{q}^{H}\right)-\mathcal{A}_{K}^{L}\left(\gamma_{q}^{K}\right)= & \int_{0}^{1} H_{t}\left(\gamma_{q}^{H}(t)\right) d t-\int u^{*} \omega \\
& -\int_{0}^{1} K_{t}\left(\gamma_{q}^{K}(t)\right) d t+\int(\bar{v} \sharp u)^{*} \omega \\
= & \int_{0}^{2}(H \sharp \bar{K})_{t}\left(\left(\gamma_{q}^{H} \sharp \gamma_{q}^{K}\right)(t)\right) d t \\
& -\int v^{*} \omega=\mathcal{A}_{H \sharp \bar{K}}\left(\gamma_{q}^{H} \sharp \gamma_{q}^{K}\right)=0
\end{aligned}
$$

by the discussion above. It remains to show that if $H$ is such that $\left.H\right|_{L}=c \in \mathbb{R}$ then $\mathcal{A}^{L}\left(\phi_{H}\right)=c$. This follows from the fact that the flow of such a Hamiltonian preserves $L$ and thus the area part of the action of any Hamiltonian chord vanishes since it is completely contained in $L$, while the first part is seen to be equal to $c$. This finishes the proof of the proposition.

2.3. The shift of spectral invariants and independence of isotopy. In this subsection, we establish the shift property of spectral invariants and use it to prove their independence of isotopy.

Theorem 2.3. Let $K, H$ be Hamiltonians such that $\phi_{K} \in \mathcal{G}_{L^{\prime}}$. Put $G_{t}=K_{t}+H_{t} \circ\left(\phi_{K}^{t}\right)^{-1}$. Then for any $A \in H F\left(L, L^{\prime}\right)$ we have

$$
\ell\left(A, G: L, L^{\prime}\right)=\ell\left(A, H: L, L^{\prime}\right)+\mathcal{A}^{L^{\prime}}\left(\phi_{K}\right) .
$$

Proof. The proof is based on the so-called naturality isomorphism, see for example $[\mathbf{2}, \mathbf{9}]$. We only sketch the construction; the interested reader is referred to these papers for details.

The map

$$
\operatorname{Crit} \mathcal{A}_{H}^{L, L^{\prime}} \rightarrow \operatorname{Crit} \mathcal{A}_{G}^{L, L^{\prime}}, \quad \gamma \mapsto \gamma^{K},
$$

where $\gamma^{K}(t)=\phi_{K}^{t}(\gamma(t))$, is well-defined and is a bijection. Perhaps the non-trivial point here is that it maps elements of $\Omega$ back to $\Omega$. This can 
be seen as follows. The curve $\gamma^{K}$ is homotopic with fixed endpoints to the concatenation $\gamma \sharp \delta$ where $\delta(t)=\phi_{K}^{t}(\gamma(1))$. But $\delta$ is a Hamiltonian chord of $K$, whose flow maps $L^{\prime}$ back to itself, and therefore it is contractible into $L^{\prime}$ by proposition 1.8. This shows that $\gamma^{K}$ is homotopic to $q_{0}$ if $\gamma$ is.

Since the path $\left\{\phi_{G}^{t}\right\}_{t}$, which is generated by $G$, is homotopic with fixed endpoints to the concatenation $\left\{\phi_{H}^{t}\right\}_{t} \sharp\left\{\phi_{K}^{t} \phi_{H}\right\}_{t}$, which is generated by the concatenated Hamiltonian $H \sharp K$, it follows from a standard argument (see for example, $[\mathbf{1 3}]$ ) that

$$
\mathcal{A}_{H \sharp K}^{L, L^{\prime}}(\gamma \sharp \delta)=\mathcal{A}_{G}^{L, L^{\prime}}\left(\gamma^{K}\right) .
$$

Since action is additive under concatenations and $\mathcal{A}_{K}^{L^{\prime}}(\delta)=\mathcal{A}^{L^{\prime}}\left(\phi_{K}\right)$ by proposition 1.8 , we have

$$
\mathcal{A}_{G}^{L, L^{\prime}}\left(\gamma^{K}\right)=\mathcal{A}_{H}^{L, L^{\prime}}(\gamma)+\mathcal{A}^{L^{\prime}}\left(\phi_{K}\right)
$$

that is, $\gamma \mapsto \gamma^{K}$ shifts the action by $\mathcal{A}^{L^{\prime}}\left(\phi_{K}\right)$.

Next we have the map

$$
\widehat{\mathcal{M}}\left(\gamma_{-}, \gamma_{+} ; H, J\right) \rightarrow \widehat{\mathcal{M}}\left(\gamma_{-}^{K}, \gamma_{+}^{K} ; G, J^{K}\right), \quad u \mapsto u^{K}
$$

where $u^{K}(s, t)=\phi_{K}^{t}(u(s, t))$ and $J_{t}^{K}=\phi_{K, *}^{t} J_{t}\left(\phi_{K, *}^{t}\right)^{-1}$. This map is a diffeomorphism. Clearly it preserves the $\mathbb{R}$-action and so passes to a diffeomorphism $\mathcal{M}\left(\gamma_{-}, \gamma_{+} ; H, J\right) \rightarrow \mathcal{M}\left(\gamma_{-}^{K}, \gamma_{+}^{K} ; G, J^{K}\right)$. The net result is that $\gamma \mapsto \gamma^{K}$ extends to a chain isomorphism

$$
C F^{a}\left(H: L, L^{\prime}\right) \rightarrow C F^{a+\mathcal{A}^{L^{\prime}}\left(\phi_{K}\right)}\left(G: L, L^{\prime}\right)
$$

for any $a$, and the assertion of the theorem follows.

Remark 2.4. Note that the proof of this theorem implies that the action spectrum $\operatorname{Spec}\left(H: L, L^{\prime}\right)$ only depends on $\phi_{H}$. This follows from the definition of the above bijection $\gamma \mapsto \gamma^{K}$, which preserves the actions if $K$ generates the identity map since then $\mathcal{A}^{L^{\prime}}\left(\phi_{K}\right)=0$.

We can now prove

Proposition 2.5. The spectral invariant $\ell\left(A, H: L, L^{\prime}\right)$ only depends on $\phi_{H} \in \mathcal{G}$.

Proof. Abbreviate $\ell(\cdot) \equiv \ell\left(A, \cdot: L, L^{\prime}\right)$. If $K$ is another Hamiltonian generating $\phi_{H}$, then the concatenation $H \sharp \bar{K}$ generates the identity map. Since $\mathcal{A}^{L^{\prime}}$ is a homomorphism to the reals, it maps the identity map to zero. It follows from theorem 2.3 that

$$
\ell(H \sharp \bar{K} \sharp K)=\ell(K)+\mathcal{A}^{L^{\prime}}\left(\phi_{H \sharp \bar{K}}\right)=\ell(K)+\mathcal{A}^{L^{\prime}}(\mathrm{id})=\ell(K) .
$$

It now suffices to note that

$$
\ell(H)=\ell(H \sharp \bar{K} \sharp K) .
$$

For a proof, see, for example [11]. 
2.4. Summary. The following theorem summarizes the various properties of spectral invariants.

Theorem 2.6. The spectral invariants descend to functions $\ell\left(A, \cdot: L, L^{\prime}\right)$ : $\mathcal{G} \rightarrow \mathbb{R} ;$ moreover, they satisfy:

(i) $\ell\left(A, \phi: L, L^{\prime}\right) \in \operatorname{Spec}(\phi) ;^{7}$

(ii) $\int_{0}^{1} \min \left(F_{t}-G_{t}\right) d t \leq \ell\left(A, \phi_{F}: L, L^{\prime}\right)-\ell\left(A, \phi_{G}: L, L^{\prime}\right) \leq \int_{0}^{1} \max \left(F_{t}-\right.$ $\left.G_{t}\right) d t$

(iii) for $\alpha \in \mathcal{G}_{L^{\prime}}$ we have $\ell\left(A, \alpha \phi: L, L^{\prime}\right)=\ell\left(A, \phi: L, L^{\prime}\right)+\mathcal{A}^{L^{\prime}}(\alpha)$;

(iv) in case $\# L \cap L^{\prime}=1$, let $A \in H F\left(L, L^{\prime}\right)=\mathbb{Z}_{2}$ be the generator; then for $\beta \in \mathcal{G}_{L}$ we have $\ell\left(A, \beta: L, L^{\prime}\right)=\mathcal{A}^{L}(\beta)$.

Proof. The only statement remaining to be proved is (iv). Let $H$ generate $\beta$. By spectrality $\ell\left(A, \beta: L, L^{\prime}\right)=\mathcal{A}_{H}^{L, L^{\prime}}(\gamma)$ where $\gamma \in \Omega$ is a Hamiltonian chord from $L$ to $L^{\prime}$. We have $\gamma(1)=\phi_{H}(\gamma(0))$. Since by assumption $\phi_{H}(L)=L$, we see that $\gamma(1) \in L$, that is $\gamma$ is a Hamiltonian chord beginning and ending on $L$. By proposition 1.8, there is a contracting half-disc $u$ for $\gamma$ into $L$. It can be seen that this $u$ may be reparametrized to produce a homotopy from $q_{0}$ to $\gamma$. Therefore

$$
\mathcal{A}_{H}^{L, L^{\prime}}(\gamma)=\mathcal{A}_{H}^{L}(\gamma)=\mathcal{A}^{L}(\beta)
$$

as claimed. The proof of the theorem is complete.

Theorem 1.9 follows from the one we just proved, therefore the main results are now proved as well.

\section{References}

[1] M. Abouzaid, A topological model for the Fukaya categories of plumbings, J. Differ. Geom. 87(1) (2011), 1-80.

[2] F. Barraud and O. Cornea, Lagrangian intersections and the Serre spectral sequence, Ann. Math. 166 (2007), 657-722.

[3] P. Biran and K. Cieliebak, Symplectic topology on subcritical manifolds, Comm. Math. Helv. 76 (2001) 712-753.

[4] Y. Chekanov, Invariant Finsler metrics on the space of Lagrangian embeddings, Math. Z. 234(3) (2000), 605-619.

[5] Y. Eliashberg and M. Gromov, Convex symplectic manifolds, in 'Several complex variables and complex geometry', Vol. 52, part 2, in Proc. Symp. in Pure Mathematics, American Mathematical Society, Providence, RI, 1991, 135-162.

[6] A. Floer, Unregularized gradient flow of the symplectic action, Commun. Pure Appl. Math. 41 (1988), 775-813.

[7] H. Hofer and D. Salamon, Floer homology and Novikov rings, in 'The Floer Memorial Volume' (A. Weinstein H. Hofer, C. Taubes, E. Zehnder, eds.), 483-524. Birkhäuser, 1995 .

\footnotetext{
${ }^{7}$ See remark 2.4 for the definition of the action spectrum $\operatorname{Spec}(\phi)$.
} 
[8] M. Khanevsky, Hofer's metric on the space of diameters, J. Topol. Anal. 1(4) (2009), 407-416.

[9] R. Leclercq, Spectral invariants in Lagrangian Floer theory, J. Mod. Dyn. 2(2) (2008), 249-286.

[10] D. McDuff, Monodromy in Hamiltonian Floer theory, Comm. Math. Helv. 85(1) (2010), 95-133.

[11] A. Monzner, N. Vichery and F. Zapolsky, Partial quasimorphisms and quasistates on cotangent bundles, and symplectic homogenization, J. Mod. Dyn. 6(2) (2012), 205-249, doi:10.3934/jmd.2012.6.205.

[12] Y.-G. Oh, Floer cohomology of Lagrangian intersections and pseudo-holomorphic disks, I, Commun. Pure Appl. Math., 46(7) (1993), 949-993.

[13] Y.-G. Oh, Construction of spectral invariants of Hamiltonian paths on closed symplectic manifolds, in The breadth of symplectic and Poisson geometry, Vol. 232 of Progress in Mathematics 525-570, Birkhäuser Boston, Boston, MA, 2005.

[14] L. Polterovich, The geometry of the group of symplectic diffeomorphisms. (Lectures in Mathematics ETH Zürich, 2001), Birkhäuser Verlag, Basel.

[15] M. Usher, Hofer's metrics and boundary depth. arXiv:1107.4599.

Mathematisches Institut DeR LMU

THERESIENSTRASSE 39

80333 Munich

Germany

E-mail address: zapolsky@yahoo.com

Received 03/10/2012, accepted 11/4/2012

I would like to thank Rémi Leclercq for helpful discussions, Leonid Polterovich for useful suggestions, and Kai Cieliebak for conversations we had as a result of which this paper came to existence. I am also grateful to the anonymous referee for remarks and corrections which allowed me to improve the exposition and rigor of the paper. Part of this work was carried out during my stay at Ludwig-Maximilians-Universität, Munich. I wish to thank this institution for a stimulating research atmosphere and hospitality. I acknowledge partial support by the DFG grant number DFG/CI45/5-1. 